\title{
Visão do acadêmico de enfermagem sobre a disciplina saúde do idoso na formação acadêmica: relato de experiência
}

\author{
The student's perception about the health assistance for the eldery patiendiscipline on the \\ academic formation: experience report
}
La visión de lós estudiantes de enfermaria la disciplina de la salud del anciano em la educación acadêmica: informe de la experiencia

Juliana Conceição Silva ${ }^{1 *}$, Cristiane Abreu da Costa ${ }^{1}$, Augusto Sergio Sousa de Oliveira ${ }^{1}$, Andreia Larissa Farias Caldas' ${ }^{1}$, Fernando Conceição de Lima ${ }^{1}$, Wanderson Renan Araújo Pinheiro', Viviane Ferraz Ferreira de Aguiar'.

\section{RESUMO}

Objetivo: Relatar a experiência vivenciada sobre a visão do acadêmico de enfermagem quanto à disciplina Saúde do Idoso em uma Instituição de Ensino Privada. Relato da Experiência: A atividade foi realizada em dois momentos: I- Roda de conversa com os acadêmicos de enfermagem e II- Realização de uma enquete. Realizaram-se seis perguntas para nortear a roda de conversa e possibilitar aos pesquisadores um diagnóstico situacional sobre o assunto, e após cada pergunta, deu-se um tempo para que os discentes abordassem seus conhecimentos e experiências com o processo de formação voltado ao idoso. Os estudantes percebem a necessidade de reorientar processo de graduação em enfermagem para lidar com o idoso; utilizam-se estratégias nas salas de aulas, tais como os casos clínicos, realização da consulta de enfermagem e simulação realística entre os alunos. Desmistificaram-se alguns conceitos vivenciados pelos discentes quanto à autonomia, independência e capacidade de realização de atividades pelos idosos. Considerações finais: resultado da pesquisa permitiu identificar que o processo de formação voltado às demandas do idoso é uma estratégia necessária que merece especial atenção das Diretrizes Curriculares Nacionais dos cursos de Bacharelado em Enfermagem para que esse seja feito de forma mais proveitosa.

Palavras-Chave: Idoso, Estudantes de enfermagem, Educação em enfermagem, Saúde do idoso.

\begin{abstract}
Objective: To report the experience about the nursing student's view on the health assistance for the eldery patient discipline. Experience Report: The activity was carried out in two moments: I- Conversation with the nursing students and II- Conducting a survey. Six questions were asked to guide the conversation circle and allow researchers a situational diagnosis about the subject, and after each question, students were given time to address their knowledge and experiences with the process of training aimed at the health. Students realize the need to reorient the undergraduate nursing process to deal with the elderly; strategies are used in the classroom, such as clinical cases, nursing consultation and realistic simulation among students. Some concepts experienced by students regarding the autonomy, independence and ability to perform activities by the elderly were demystified. Final considerations: The result of the research identified that the training process focused on the demands of the elderly is a necessary strategy that deserves special attention from the National Curriculum Guidelines of the Bachelor of Nursing courses so that it can be done more profitably.
\end{abstract}

Key words: Aged, Students, Nursing, Education, Health of the elderly.

\section{RESUMEN}

Objetivo: Informar la experiencia vivida sobre la opinión del estudiante de enfermería sobre el tema de la Salud de los Ancianos en una institución de educación privada. Informe de experiencia: La actividad se

${ }^{1}$ Centro Universitário Metropolitano da Amazônia (UNIFAMAZ), Belém-Pará.

*E-mail: Juliana.cs.18@hotmail.com

SUBMETIDO EM: 9/2019

ACEITO EM: 10/2019

PUBLICADO EM: 1/2020

REAS/EJCH |Vol.Sup.38 | e1842 | DOI: https://doi.org/10.25248/reas.e1842.2020 Página 1 de 6 
realizó en dos momentos: I- Conversación con los estudiantes de enfermería y II- Realización de una encuesta. Se hicieron seis preguntas para guiar el círculo de conversación y permitir a los investigadores un diagnóstico situacional sobre el tema, y después de cada pregunta, los estudiantes tuvieron tiempo para abordar sus conocimientos y experiencias con el proceso de capacitación dirigido a los ancianos. Los estudiantes se dan cuenta de la necesidad de reorientar el proceso de pregrado de enfermería para tratar con los ancianos; Las estrategias se utilizan en el aula, como casos clínicos, consulta de enfermería y simulación realista entre los estudiantes. Se desmitificaron algunos conceptos experimentados por los estudiantes con respecto a la autonomía, independencia y capacidad para realizar actividades de los ancianos. Consideraciones finales: El resultado de la investigación identificó que el proceso de capacitación centrado en las demandas de las personas mayores es una estrategia necesaria que merece atención especial de las Directrices Curriculares Nacionales de los cursos de Licenciatura en Enfermería para que pueda realizarse de manera más rentable.

Palabras clave: Anciano, Estudiantes de enfermería, Educación en enfermería, Salud del anciano.

\section{INTRODUÇÃO}

O envelhecimento mundial da população tem sido motivado principalmente por mudanças nas condições de vida e avanços da medicina que reduziram taxas de mortalidade precoce e aumentaram a expectativa de vida. Desse modo, observa-se que desde a década de 1950, a população ganhou cerca de 22,5 anos e se estima que nos últimos 5 anos uma percentual expressivo de pessoas alcançou a margem de idades superiores a 74,3 anos (SENA LB, et al., 2016).

Com o aumento do número de idosos tem-se discutido sob o campo da geriatria e gerontologia, na busca da promoção da saúde, tratamento de doenças e incapacidades entre os idosos com intuito de investigar os aspectos clínicos, biológicos, psicológicos, sociais, culturais, econômicos e históricos (CARVALHO CRA, 2015). Mendes EV (2015) evidencia que a Enfermagem deve realizar ações efetivas sustentadas pela cientificidade da profissão, visto a crescente necessidade por ações e serviços de saúde voltados à pessoa idosa, na perspectiva de promover ações singulares de saúde, resolutivas que compreendam uma linha de cuidado integral.

Nesse contexto, a abordagem sobre a saúde do idoso na grade curricular do acadêmico de enfermagem é necessária, pois proporciona o desenvolvimento de competências em múltiplas dimensões como fisiologia e fisiopatologia do envelhecimento humano, conduta resolutiva diante das principais demandas ofertadas pelos idosos, concepção de saúde resolutiva na sua dimensão biopsicossocial e não apenas como um distúrbio biológico, aspectos éticos e o relacionamento com a equipe multiprofissional, numa perspectiva de integralidade (CARVALHO CRA e HENNINGTON EA, 2015). No Brasil, a Política Nacional de Saúde da Pessoa Idosa (PNSPI) reforça a necessidade de adequar currículos e inserir em diversos níveis de ensino formal, conteúdos voltados ao processo do envelhecimento (CARVALHO CRA, 2015).

Silva M (2010) e Xavier AS e Koifman L (2011) inferem que em algumas instituições o ensino nesta área é ofertado em modalidades à distância, de forma online ou como disciplina optativa, dificultando, assim, o contato com este público-alvo e o desenvolvimento de habilidades e competências a serem desenvolvidas no atendimento ao idoso. A falta de contato dos discentes com a disciplina, em seus currículos, promove atitudes desfavoráveis em relação à conduta voltada ao idoso (MONTANHOLI L et al., 2006).

Esta pesquisa justifica-se devido à necessidade de instigar as Instituições de Ensino superior a oferecer, dentro do Projeto Pedagógico, a disciplina saúde do idoso de cunho teórico, prático e que seja presencial, pois devido ao aumento do número de idosos há necessidade de profissionais de saúde qualificados. Sendo assim, o objetivo desta pesquisa foi relatar uma experiência vivenciada sobre a visão do acadêmico de enfermagem quanto à disciplina Saúde do Idoso em uma Instituição de Ensino Privada.

\section{RELATO DA EXPERIÊNCIA}

Trata-se de um relato de experiência, realizado pelos acadêmicos de enfermagem do sexto período de uma Instituição de Ensino Privada, no município de Belém-Pará, no mês de maio de 2018. 
A escolha da temática emergiu a partir da vivência com o processo de formação nas aulas de Enfermagem na Atenção à Saúde do Idoso. Para o conhecimento científico a respeito do tema, de início levantou-se na literatura uma busca por materiais bibliográficos que abordassem sobre o processo de formação voltado à saúde do idoso. Com os achados alcançados, os pesquisadores tiveram melhor embasamento para definir o problema de pesquisa a ser estudado e a metodologia que melhor se adequava para responder a este objetivo outrora proposto. Além disso, utilizou-se como problema de pesquisa: "O aumento do número de idosos e a fragilidade do processo de formação voltado especificamente para lidar com a saúde do idoso".

O público alvo foi composto por 14 discentes do sétimo semestre, do curso de bacharelado em enfermagem de uma Instituição de Ensino Superior Privada que já passaram pela disciplina de "Enfermagem na Atenção à Saúde do Idoso e experimentaram a grande curricular e o plano de ensino da disciplina. $O$ cenário da ação foi o próprio ambiente universitário, pelos alunos terem maior comodidade e inspiração para poder discutir sobre o assunto.

A atividade foi realizada em dois momentos principais: I- Roda de conversa com os acadêmicos de enfermagem e II- Realização de uma enquete. Foram realizadas seis perguntas para nortear a roda de conversa e possibilitar aos pesquisadores um diagnóstico situacional sobre o assunto na perspectiva dos participantes, e após cada pergunta foi dado um tempo para que os discentes abordassem seus conhecimentos e experiências com o processo de formação voltado para atender as necessidades dos idosos.

As perguntas que nortearam a conversa foram: "Você considera a disciplina saúde do idoso importante para a formação acadêmica?" Quanto ao nível de aproveitamento, sua experiência com a disciplina foi boa? Qual a sua perspectiva em relação ao processo de envelhecimento ates e após o contato com a disciplinas? A carga horária-aula ofertada pela disciplina foi satisfatória? Vocês sentiram necessidade de aula prática externa para fazer o intercâmbio com a teoria dada em sala de aula, seja na Atenção Básica como na rede Hospitalar? Como você entende o cuidado de enfermagem no processo do envelhecimento? Vocês já atenderam um usuário idoso durante uma consulta de enfermagem, se sim, houve algum tipo de dificuldade ao consultá-lo?"

No segundo momento foi aplicada uma enquete que continha cinco perguntas, com alternativas de "SIM" ou "NÃO", a saber: "A disciplina saúde do idoso mudou sua visão quanto ao processo de envelhecimento?", "Você sabe a diferença entre senilidade e senescência?", "A enfermagem tem contribuído na abordagem do cuidado em aspectos do processo de envelhecimento?", "A enfermagem desempenha papel importante na execução e cumprimento das leis direcionadas aos idosos, promovendo a inclusão social indiscriminada dos idosos, respeitando suas necessidades, capacidades e limitações?" Você já teve a oportunidade de realizar ou participar da consulta de enfermagem ao paciente idoso na atenção básica ou hospitalar?

Da experiência vivenciada emergiram duas categorias: Conversando em roda: conhecimento e experiência dos acadêmicos de enfermagem na disciplina Saúde do Idoso e Processo do envelhecimento e as alterações na senescência e senilidade.

Na primeira categoria percebeu-se que os estudantes entendem que é necessário abordar sobre a temática de saúde do idoso em disciplinas presenciais com dinamismo, de forma crítica-reflexiva sustentada por uma estratégia dinâmica, pois o aumento do número de idosos e suas particularidades devem ser identificados e avaliados conforme as necessidades dos idosos. Salienta-se, pelos graduandos que a disciplina permitiu uma reflexão sobre as alterações fisiológicas e patológicas comum aos idosos, a promoção de um envelhecimento ativo e saúde, utilização de instrumentos de avaliação geriátrica na consulta de enfermagem e a preocupação com as novas demandas que têm sido destacadas na área da gerontogeriatria. O desenvolvimento de competências e habilidades são identificadas, contudo, a disciplina ainda apresenta uma carga horária baixa, associada a redução de aulas práticas externas e estágio obrigatório. Entre as estratégias utilizadas na sala de aula estão os casos clínicos, realização da consulta de enfermagem e simulação realística entre os próprios alunos.

$\mathrm{Na}$ segunda categoria enquete permitiu a identificaçãode que os discentes entendem que a identificação precoce da vulnerabilidade da vulnerabilidade do idoso deve ser realizada. A comparação entre o antes e 
depois da disicplina desmistificou alguns conceitos vivenciados pelos discentes sobre o processo de envelhecimento, evidenciando que o idoso pode e deve ser autônomo, independente com manutençã da funcionalidade e ter uma vida ativa e saudável, conforme a Figura 1.

Figura 1 - Visão dos discentens de enfermagem antes e após a disciplina saúde do idoso, 2018.

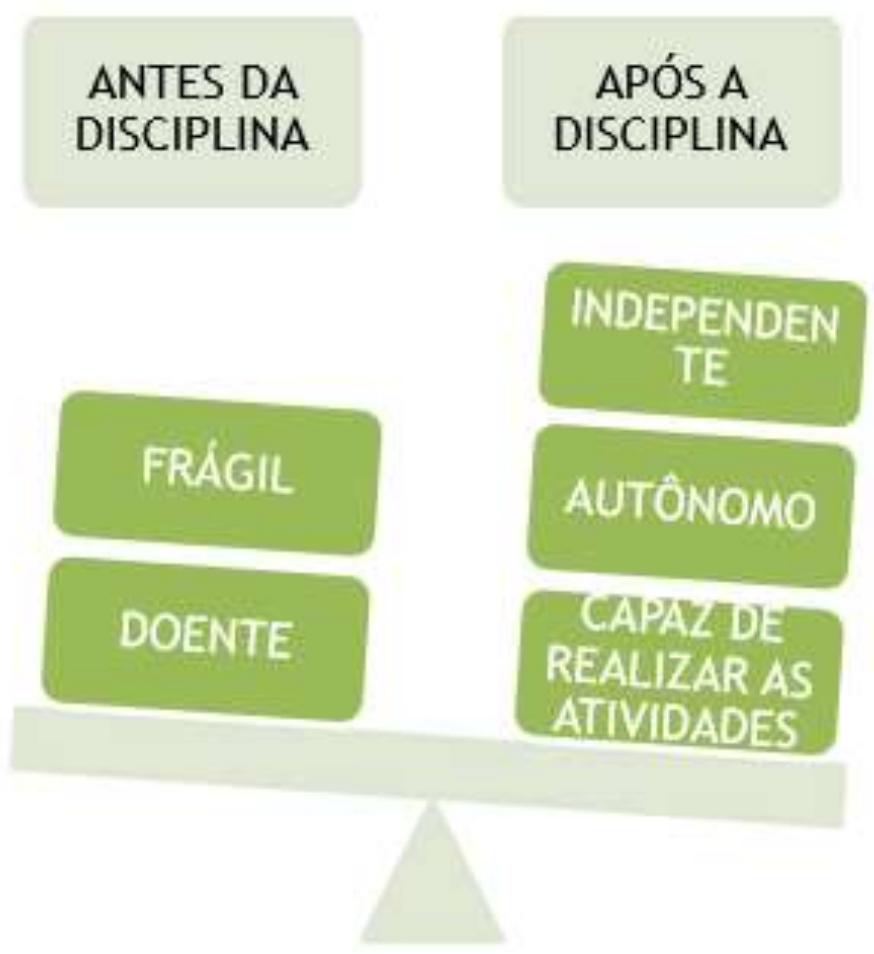

Fonte: Silva et al., 2018.

\section{DISCUSSÃO}

Faz-se necessário refletir sobre os fatores fisiopatológicos que frequentemente acometem aos idosos, bem como a importância do envelhecimento ativo para que os profissionais desenvolvam suas práticas com vista a promover o completo bem-estar biopsicossocial desses usuários (MALLMANN DG, et al., 2015). O envelhecimento ativo e saudável e que permita melhor qualidade de vida deve ser garantido ao idoso mediante ao crescimento expressivo de idosos no presente cenário mundial, nesta perspectiva, deve-se inovar e readequar as ações de saúde que promovam um envelhecimento saudável (AGUIAR VFF, et al., 2019).

Neste contexto, a disciplina voltada a saúde do idoso deve permitir não somente o conhecimento pelo aluno, mas também a experiência por meio da prática, a partir da aproximação desses idosos. A convivência em espaços diferentes do habitual, como as aulas práticas extramuros contribuem substancialmente para o processo de ensino-aprendizagem, visto que algumas mudanças no modo de interagir, representar o pensamento, emoções, além de produzir e compartilhar conhecimentos deve acontecer e são necessárias. Assim, novas experiências podem ser somadas com aquelas da sala de aula e assim possibilitar ao aluno conteúdos para ajudar na sua prática profissional (VALENTE JÁ, et al., 2017).

Além disso, Benetti $P$, et al. (2015) relatam em seu estudo que a prática de extensão universitária proporciona na formação do estudante uma experiência proveitosa em que se é possível realizar o intercâmbio entre a teoria e a prática e, por isso, devem estar incumbidas nos currículos da graduação, pois permite uma ação transformadora, que influencia diretamente no atendimento dos interesses e necessidades 
especificas da população. Quanto ao Estágio Curricular Supervisionado (ECS) promove o convívio com a rotina do trabalho, permitindo o desenvolvimento de conhecimentos e habilidades pertinentes à prática profissional, bem como o pensamento crítico e raciocínio clínico necessário para a construção do perfil profissional (OLIVEIRA CPS, et al., 2017).

O enfermeiro tem como uma de suas atribuições o cuidado na prevenção e promoção da saúde dos idosos, pois este tem seu sistema imunológico, cardiovascular, respiratório e articular diminuídos fisiologicamente. (SILVA NA, et al., 2015). Registra-se, desta maneira, a consulta de enfermagem como uma ação especifica deste profissional (MARANHA NB, et al., 2017), que deve constar no desenvolvimento da assistência de enfermagem em qualquer nível de saúde, quer seja em ambiente públicos ou privados (DANTAS CN, et al., 2016), tal prática propicia a estruturação do vínculo entre profissional e usuário além da avaliação no aspecto biopsicossocial como forma de cuidado integral (KAHL C, et al., 2018).

A utilização de metodologias de ensino- aprendizagem devem ser utilizadas. De acordo com Ferreira RP, et al (2018) a utilização da simulação realística na formação em enfermagem pode produzir impactos positivos sobre o processo de aprendizagem, haja vista que contribui positivamente para a efetivação do vínculo entre a teoria e a prática e permite o aprendizado significativo, contribuindo para o crescimento profissional. Pontuase que a compreensão que os profissionais de saúde têm dos idosos interfere na maneira de assisti-los e tratá-los (REIS FFS, et al., 2017).

Quanto ao processo de envelhecimento o idoso pode ser acometido por alterações voltadas as aspectos biológicos, psicológicos, sociais e culturais que podem fragilizar sua experiência nessa fase da vida. (AGUIAR VFF, et al., 2019). Por esta razão, é necessário a estratigficação de risco para que se identifique de forma precoce a vulnerabilidade para que se possa tomar a conduta mais adequada. (MORAES EN, 2016). A senescência e a senilidade, de acordo com Mesquita JS, et al. (2016) necessitam de atenção dos profissionais de saúde para lidar com a demanda específica de cada idoso, sendo a promoção da saúde à pessoa idosa uma vertente diante das comorbidades que Ihe possam acometer.

O resultado da pesquisa permitiu identificar que o processo de formação voltado a atender integralmente as demandas da pessoa idosa é uma estratégia necessária que merece especial atenção das Diretrizes Curriculares Nacionais dos cursos de Bacharelado em Enfermagem para que esse seja feito de forma mais proveitosa. O resultado da ação identificou fatores que precisam ser analisados, tal como a redução da carga horária prática, visto que tal processo é um facilitador para o entendimento e ajudaria na futura atuação profissional. Infere-se que a ação permitiu aos graduandos um olhar mais crítico para a atual realidade. Destaca-se, ainda, como limitação do estudo a falta de acompanhamento por mais tempo das futuras turmas que ingressarão no curso de enfermagem para avaliar a atual situação. Assim, espera-se que os resultados aqui encontrados possam estimular pesquisadores, gestores, docentes, coordenadores e os próprios acadêmicos na busca de estratégias que permitam o fortalecimento do processo de formação em enfermagem voltado para atender ao idoso.

\section{REFERÊNCIAS}

1. SILVA NA, et al. Desempenho funcional e sua associação com variáveis antropométricas e de composição corporal em idosos. Ciênc. saúde coletiva, 2015; 20(12): 3723-3732.

2. MALLMANN DG, et al. Educação em saúde como principal alternativa para promover a saúde do idoso. Ciênc. saúde coletiva, 2015; 20(6): 1763-1772.

3. AGUIAR VFF, et al. Intergenerationality between the elderly and adolescents in the search for the deconstruction of stereotypes in old age: experience report. Revista Eletrônica Acervo Saúde, 2019; (23): 413.

4. CARVALHO CRA. "A 'saúde do idoso' no ensino superior de universidades públicas do Rio de Janeiro: o caso dos cursos de Educação Física, Enfermagem e Nutrição”. Tese (Doutorado) - Escola Nacional de Saúde Pública Sergio Arouca, Rio de Janeiro, 2015.

5. CARVALHO CRA, HENNINGTON ÉA. A abordagem do envelhecimento na formação universitária dos profissionais de saúde: uma revisão integrativa. Rev. Bras. Geriatr. Gerontol, 2015; 18(2): 417-431. 
6. MONTANHOLI LL et al. Ensino sobre idoso e gerontologia: Visão do discente de enfermagem no estado de Minas Gerais. Texto contexto enferm, 2006; 15(4): 663-71.

7. OLIVEIRA CPS et al. O estágio curricular supervisionado II como potencializador na formação de acadêmicos de enfermagem: relato de experiência. In: INTERNATIONAL NURSING CONGRESS, 2017; Vancouver Theme: Good practices of nursing representations In the construction of Society.

8. REIS FFS et al. Conhecimento da equipe de enfermagem de um hospital público sobre o envelhecimento humano. Revista de enfermagem UFPE online, 2017; 11(6): 2594-603.

9. SENA LB et al. Conhecimento do enfermeiro sobre políticas de saúde da pessoa idosa. Revista de enfermagem UFPE online, 2016; 10(3): 1459-65.

10. SILVA M. Educar na cibercultura: Desafios à formação de professores para docência em cursos online. Revista digital de tecnologias cognitivas, 2010; 3: 36-51.

11. AGUIAR VFF, et al. Ação educativa com profissionais de saúde na identificação do idoso vulnerável: relato de experiência. Revista Eletrônica Acervo Saúde, 2019;(28): 932.

12. VALENTE JÁ, et al. Metodologias ativas: das concepções às práticas em distintos níveis de ensino. Revista diálogo educacional, 2017; 17(52): 455-478.

13. MORAES EN et al. Índice de Vulnerabilidade Clínico Funcional-20 (IVCF-20): reconhecimento rápido do idoso frágil. Rev Saúde Pública, 2016; 50:81.

14. XAVIER AS; KOIFMAN L. Educação superior no Brasil e a formação dos profissionais de saúde com ênfase no envelhecimento. Interface-Comunicação, Saúde, Educação, 2011; 15(39): 973-984.

15. MENDES EV. A construção social da atenção primária à saúde. Brasilia: CONASS; 2015; vol 1, 1999 p.

16. BENETTI P, et al. CREDITAÇÃO DA EXTENSÃO UNIVERSITÁRIA NOS CURSOS DE GRADUAÇÃO: RELATO DE EXPERIÊNCIA. REVISTA BRASILEIRA DE EXTENSÃO UNIVERSITÁRIA, 2015. 6(1): 25-32.

17. KAHL C, et al. Actions and interactions in clinical nursing practice in Primary Health Care. Rev Esc Enferm USP, 2018;52: 03327.

18. FERREIRA RP, et al. Simulação Realística como Estratégia de Ensino no Aprendizado de Estudantes da Área da Saúde. Revista de Enfermagem do Centro-Oeste Mineiro, 2018; 8:2508.

19. MESQUITA JS, et al. Promoção da saúde e integralidade na atenção ao idoso: uma realidade brasileira? Revista Kairós: Gerontologia, [S.I.], 2016; 19(1): 227-238.

20. DANTAS CN, et al. a consulta de enfermagem como tecnologia do cuidado à luz dos pensamentos de bacon e galimberti. Texto contexto - enferm, 2016; 25(1):e2800014.

21. MARANHA NB, et al. A consulta de enfermagem no cenário da atenção básica e a percepção dos usuários: revisão integrativa. Academus Revista Científica da Saúde, 2017;2(1). 